Pacific Journal of Mathematics

DIFFERENCE EQUATIONS FOR SOME ORTHOGONAL

H. L. Rall AND I. M. ShefFe 


\title{
DIFFERENCE EQUATIONS FOR SOME ORTHOGONAL POLYNOMIALS
}

\author{
H. L. Krall and I. M. Sheffer
}

It is well-known that every orthogonal polynomial set $\left\{P_{n}(x)\right\}$ satisfies a 3 -term recurrence relation of the form

$$
P_{n+1}(x)=\left(a_{n} x+b_{n}\right) P_{n}(x)+c_{n} P_{n-1}(x) \quad(n=1,2, \cdots) .
$$

Some orthogonal sets (polynomials of Jacobi, Hermite and so on) are solutions of differential equations. It will be shown that there exist orthogonal polynomial sets that satisfy 3 -term difference equations of the form

$$
A(x) y(x+\alpha)+B(x) y(x-\alpha)+C(x) y(x)=\lambda y(x)
$$

where $A, B, C$ are polynomials of degree $\leqq 2$ and $\lambda$ is a parameter.

Consider the difference equation

$$
A(x) y(x+\alpha)+B(x) y(x+\beta)+C(x) y(x)=\lambda y(x)
$$

where $A, B, C$ are real polynomials, $\lambda$ is a parameter, and $\alpha, \beta, 0$ are distinct and real. We examine two cases, according as $A, B, C$ are of degree $\leqq 1$ :

(a) $A(x)=a_{1} x+a_{0}, B(x)=b_{1} x+b_{0}, C(x)=c_{1} x$, or are of degree $\leqq 2$ :

(b) $A(x)=a_{2} x^{2}+a_{1} x+a_{0}, B(x)=b_{2} x^{2}+b_{1} x+b_{0}, C(x)=c_{2} x^{2}+c_{1} x$ $\left(a_{2}, b_{2}, c_{2}\right.$ not all zero). We shall use the notation (1.3a), (1.3b) to denote equation (1.3) for the respective conditions (a), (b).

Equation (1.3) will be termed admissible if there exists a real sequence $\left\{\lambda_{n}\right\}(n=0,1, \cdots)$ such that for $\lambda=\lambda_{n}$ there is a polynomial solution $y_{n}(x)$, unique to within a multiplicative constant, and $y_{n}(x)$ is of degree (exactly) $n$. It follows that admissibility implies that

$$
\lambda_{m} \neq \lambda_{n}(m \neq n) \text {. }
$$

Lemma 1.1. Equation (1.3a) is admissible if and only if

$$
a_{1}+b_{1}+c_{1}=0, \quad \delta \equiv a_{1} \alpha+b_{1} \beta \neq 0 .
$$

And in this case we have

$$
\lambda_{n}=\left(a_{0}+b_{0}\right)+n\left(a_{1} \alpha+b_{1} \beta\right) \quad(n=0,1, \cdots) .
$$

Proof. Let $n$ be arbitrary. If we substitute the $n$th degree polynomial 


$$
y(x)=x^{n}+\sum_{j=0}^{n-1} p_{j} x^{j}
$$

into (1.3a), a necessary and sufficient condition that $y(x)$ be a solution is that coefficients of $x^{n+1}, x^{n}, \cdots$ agree on both sides. The coefficient of $x^{n+1}$ yields the first of (1.5), that of $x^{n}$ gives $\lambda=\lambda_{n}$ as in (1.6); and those of $x^{n-1}, \cdots, x^{0}$ give successive equations for $p_{n-1}, \cdots, p_{0}$. In these equations the coefficients of $p_{n-1}, \cdots, p_{0}$ are respectively $\lambda_{n}-\lambda_{n-1}, \lambda_{n}-\lambda_{n-2}, \cdots, \lambda_{n}-\lambda_{0}$, so there is one and only one choice of the $p_{\jmath}$ 's if and only if $\lambda_{n} \neq \lambda_{j}(j \leqq n-1)$. This condition is equivalent to the second part of (1.5); and the lemma is established.

Lemma 1.2. Equation (1.3b) is admissible if and only if

$$
\begin{array}{ll}
a_{2}+b_{2}+c_{2}=0, \quad a_{1}+b_{1}+c_{1}=0, & a_{2} \alpha+b_{2} \beta=0 ; \\
2\left(a_{1} \alpha+b_{1} \beta\right)+n\left(a_{2} \alpha^{2}+b_{2} \beta^{2}\right) \neq 0 & (n=0,1, \cdots) .
\end{array}
$$

And in this case $\lambda_{n}$ is given by

$$
\begin{aligned}
\lambda_{n} & =\left(a_{0}+b_{0}\right)+n\left(a_{1} \alpha+b_{1} \beta\right)+n(n-1)\left(a_{2} \alpha^{2}+b_{2} \beta^{2}\right) / 2 \\
(n & =0,1, \cdots) .
\end{aligned}
$$

Proof. Substituting (1.7) into (1.3b) and equating like terms (as a necessary and sufficient condition for a solution) we find that the terms in $x^{n+2}, x^{n+1}$ give (1.8), the $x^{n}$ term gives $\lambda=\lambda_{n}$ as in (1.10), and $p_{n-1}, \cdots, p_{0}$ again are uniquely determined if and only if $\lambda_{n} \neq \lambda_{j}$ $(j \leqq n-1)$. Now the condition $\lambda_{m} \neq \lambda_{n}(m \neq n)$ is seen to reduce to $(1.9)$; so the lemma is proved.

In the proofs of Lemmas $1.1,1.2$ it was seen that if a polynomial $y(x)$ of degree $n$ satisfies (1.3a or b) then the corresponding value of $\lambda$ is $\lambda_{n}$ as given by (1.6) or (1.10); so we have the

Corollary. If (1.3a) or (1.3b) is admissible then for each $\lambda \neq \lambda_{n}$ $(n=0,1, \cdots)$ the only polynomial solution is $y(x) \equiv 0$.

Let $(1.3 \mathrm{a})$ or $(1.3 \mathrm{~b})$ be admissible. In both cases the solution for $n=1$ is

$$
y_{1}(x)=x+\left(a_{0} \alpha+b_{0} \beta\right) \delta^{-1}
$$

where $\delta$ is given in (1.5). If we set

$$
x+d=x^{*}, z\left(x^{*}\right)=y\left(x^{*}-d\right)
$$

with

$$
d=\left(a_{0} \alpha+b_{0} \beta\right) \delta^{-1},
$$


the equation in $z\left(x^{*}\right)$ will also be admissible and will have the form (1.3a) or (1.3b) after the constant term in $C\left(x^{*}\right)$ has been absorbed into the $\lambda$. Moreover, for $n=1$ we have

$$
z_{1}\left(x^{*}\right)=x^{*} \text {. }
$$

An admissible equation (1.3a) or (1.3b) in which for $n=1$ the solution contains no constant term will be called canonical. It is no restriction to limit ourselves to canonical equations.

From (1.11) we obtain

Lemma 1.3. The admissible equation (1.3a) or (1.3b) is canonical if and only if

$$
a_{0} \alpha+b_{0} \beta=0
$$

2. Orthogonality for case (1.3a). We consider the problem of determining those canonical equations (1.3a) [(1.3b) in $\S 3]$ whose polynomial solutions form an orthogonal set. For all polynomials $y(x)$ we have

$$
y(x+u)=\sum_{k=0}^{\infty} y^{(k)}(x) u^{k} / k !
$$

so (1.3a) is equivalent, with respect to polynomial solutions, to the differential equation of infinite order

$$
x y^{\prime}(x)+\sum_{k=2}^{\infty} H_{k}(x) y^{(k)}(x) / k !=\sigma y(x)
$$

where

$$
H_{k}(x)=r_{k}+s_{k} x=\left(a_{0} \alpha^{k}+b_{0} \beta^{k}\right) \hat{\delta}^{-1}+\left(a_{1} \alpha^{k}+b_{1} \beta^{k}\right) \delta^{-1} x
$$

$(k=1,2, \cdots)$ with $\sigma=\left\{\lambda-\left(a_{0}+b_{0}\right)\right\} \hat{o}^{-1}$. Using (1.6) we find that the sequence $\left\{\sigma_{n}\right\}$ for which there are polynomial solutions is given by $\sigma_{n}=n$.

Equation (2.2) is identical with equation (3.1) of [1]. In Remark (i) ([1], p. 151) it is shown that if $r_{2}=0$ the polynomial solutions do not form an orthogonal set. We therefore assume $r_{2} \neq 0$. In this case, Theorem 3.1 ([1], p. 151) states that the solutions of (our present) equation (2.2), hence of cononical equation (1.3a), form a weak orthogonal set if and only if

$$
\begin{array}{ll}
r_{2 p+1}=0, & s_{2 p+1}=s_{s}^{p}, \\
r_{2 p+2}=r_{2} s_{3}^{p}, & s_{2 p+2}=s_{2} s_{3}^{p}
\end{array} \quad(p=0,1, \cdots) .
$$

Moreover the weak orthogonal set is an orthogonal set when and only when one of the following two relations holds: 


$$
\begin{aligned}
& s_{2}^{2}-s_{3}=0 ; \\
& s_{2}^{2}-s_{3} \neq 0 \text { and } 2 r_{2}\left(s_{2}^{2}-s_{3}\right)^{-1} \neq 0,1,2, \cdots .
\end{aligned}
$$

The condition $r_{2 p+1}=0$ is

$$
a_{0} \alpha^{2 p+1}+b_{0} \beta^{2 p+1}=0 \quad(p=0,1, \cdots) .
$$

If $a_{0}=0$ or $b_{0}=0$ then both are zero since $\alpha \beta \neq 0$. But then $r_{2}=0$, contrary to assumption. So $a_{0} b_{0} \neq 0$. Taking $p=0,1$ in (2.6) we then get $\beta^{2}=\alpha^{2}$. Since $\alpha, \beta$ are distinct, then $\beta=-\alpha$; and again from (2.6) with $p=0: a_{0}=b_{0}$. Thus, if $r_{2} \neq 0$ then $r_{2 p+1}=0(p=0,1, \cdots)$ if and only if

$$
\beta=-\alpha, a_{0}=b_{0} \neq 0 .
$$

With (2.7) holding then

$$
\delta=\alpha\left(a_{1}-b_{1}\right) \neq 0,
$$

so

$$
\begin{aligned}
& r_{2 p+1}=0, s_{2 p+1}=\alpha^{2 p}, r_{2 p+2}=2 a_{0}\left(a_{1}-b_{1}\right)^{-1} \alpha^{2 p+1}, \\
& s_{2 p+2}=\left(a_{1}+b_{1}\right)\left(a_{1}-b_{1}\right)^{-1} \alpha^{2 p+1} .
\end{aligned}
$$

Conditions (2.4) are seen to be satisfied. And $\left(2.5_{1}\right),\left(2.5_{2}\right)$ become respectively:

$$
\begin{aligned}
& a_{1} b_{1}=0 ; \\
& a_{1} b_{1} \neq 0, a_{0}\left(a_{1}-b_{1}\right)\left(\alpha a_{1} b_{1}\right)^{-1} \neq 0,1, \cdots .
\end{aligned}
$$

To sum up:

Theorem 2.1. Let equation (1.3a) be canonical. Then its polynomial solutions from an orthogonal set if and only if (2.7) holds and one of $\left(2.9_{1}\right),\left(2.9_{2}\right)$ holds.

REMARKs. ( i ) If (1.3a) is canonical its polynomial solutions form an orthogonal set if and only if it is of the form

$$
\begin{aligned}
\left(a_{1} x+a_{0}\right) y(x+\alpha) & +\left(b_{1} x+a_{0}\right) y(x-\alpha) \\
& -\left(a_{1}+b_{1}\right) x y(x)=\lambda y(x),
\end{aligned}
$$

with $a_{0} \neq 0, a_{1} \neq b_{1}, \alpha \neq 0$, and either (2.9 $)$ or (2.9 $)$ holding.

(ii) In (2.10) make the variable changes $x=\alpha x^{*}, z\left(x^{*}\right)=y\left(\alpha x^{*}\right)$. There results a similar difference equation in $z\left(x^{*}\right)$, in which $\alpha$ is replaced by 1 . This equation has an orthogonal set of solutions when (2.10) does. It may be termed a standard canonical equation. After 
dividing by $a_{0}$ this equation has the form (dropping asterisks)

$$
\begin{aligned}
\left(c_{1} x+1\right) z(x+1) & +\left(d_{1} x+1\right) z(x-1) \\
& -\left(c_{1}+d_{1}\right) x z(x)=\mu z(x),
\end{aligned}
$$

with $c_{1}-d_{1} \neq 0$ and either $c_{1} d_{1}=0$ or

$$
c_{1} d_{1} \neq 0,\left(c_{1}-d_{1}\right)\left(c_{1} d_{1}\right)^{-1} \neq 0,1,2, \cdots \text {. }
$$

3. Orthogonality for case (1.3b). Let equation (1.3b) be canonical, so that (1.12) holds. Putting (2.1) into (1.3b) we get an infinite order differential equation with polynomial coefficients of degree $\leqq 2$, which is equivalent to $(1.3 \mathrm{~b})$ at least for polynomial solutions:

$$
x y^{\prime}(x)+\sum_{k=2}^{\infty} T_{k}(x) y^{(k)}(x) / k !=\sigma y(x)
$$

where

$$
\begin{aligned}
T_{k}(x)= & r_{k}+s_{k} x+t_{k} x^{2}=\left(a_{0} \alpha^{k}+b_{0} \beta^{k}\right) \delta^{-1}+\left(a_{1} \alpha^{k}+b_{1} \beta^{k}\right) \delta^{-1} x \\
& +\left(a_{2} \alpha^{k}+b_{2} \beta^{k}\right) \delta^{-1} x^{2} \quad(k=2,3, \cdots)
\end{aligned}
$$

and $\sigma=\left\{\lambda-\left(a_{0}+b_{0}\right)\right\} \delta^{-1}$ and $\delta$ is given by (1.5). From (1.10) we see that $\left\{\sigma_{n}\right\}$ is given by

$$
\sigma_{n}=n+n(n-1) t_{2} / 2 .
$$

Equations of the form (3.1), that is, with $\max _{k}$ \{degree $\left.T_{k}(x)\right\}=2$ were considered in [1], but the results obtained were not as complete as for the case where the coefficients are of degree $\leqq 1$. We must therefore proceed differently. We first show that if canonical equation (1.3b), hence also (3.1), has an orthogonal set of solutions then $\beta=-\alpha$.

For suppose not. Then $|\alpha| \neq|\beta|$, since $\alpha, \beta$ are distinct. We may assume that $|\alpha|>|\beta|$. By Theorem 2.2 ([1], p. 148) there is a sequence of constants $\left\{\alpha_{n}\right\}$ (the moments of the weight function corresponding to the orthogonal set), with $\alpha_{0} \neq 0$, that satisfies the system of equations

$$
d_{p+k}^{p}=0, D_{p+k}^{p}=0 \quad(p, k=0,1, \cdots)
$$

where (in our present case, as seen in [1], p. 153)

$$
\begin{aligned}
& d_{p+k}^{p}=\sum_{i=k}^{2 k+2} \alpha_{i}\left[\left(\begin{array}{c}
k \\
i-k
\end{array}\right) r_{2 p+2 k+1-i}+\left(\begin{array}{c}
k \\
i-k-1
\end{array}\right) s_{2 p+2 k+2-i}\right. \\
& \left.+\left(\begin{array}{c}
k \\
i-k-2
\end{array}\right) t_{2 p+2 k+3-i}\right] \text {, }
\end{aligned}
$$




$$
\begin{aligned}
D_{p+k}^{p}= & \sum_{i=k}^{2 k+3} \alpha_{i}\left[\frac{i+1}{k+1}\left(\begin{array}{c}
k+1 \\
i-k
\end{array}\right) r_{2 p+2 k+2-i}\right. \\
& +\frac{i}{k+1}\left(\begin{array}{c}
k+1 \\
i-k-1
\end{array}\right) s_{2 p+2 k+3-i} \\
& \left.+\frac{i-1}{k+1}\left(\begin{array}{c}
k+1 \\
i-k-2
\end{array}\right) t_{2 p+2 k+4-i}\right] .
\end{aligned}
$$

Here the convention is made that $\left(\begin{array}{c}m \\ q\end{array}\right)=0$ for $q<0$, and $r_{j}=s_{j}=$ $t_{j}=0$ for $j \leqq 0$ and $r_{1}=t_{1}=0, s_{1}=1$.

Putting the values of $r_{k}, s_{k}, t_{k}$ from (3.2) into (3.3) we get

$$
\left\{\begin{array}{l}
\alpha^{2 p+2 k+1} U_{k}+\beta^{2 p+2 k+1} V_{k}=0 \\
\alpha^{2 p+2 k+2} W_{k}+\beta^{2 p+2 k+2} X_{k}=0
\end{array} \quad(p, k=0,1, \cdots)\right.
$$

where

$$
\begin{aligned}
U_{k}= & \sum_{i=k}^{2 k+2} \alpha_{i}\left[\left(\begin{array}{c}
k \\
i-k
\end{array}\right) a_{0} \alpha^{-i}+\left(\begin{array}{c}
k \\
i-k-1
\end{array}\right) a_{1} \alpha^{-i+1}\right. \\
& \left.+\left(\begin{array}{c}
k \\
i-k-2
\end{array}\right) a_{2} \alpha^{-i+2}\right] \\
W_{k}= & \sum_{i=k}^{2 k+3} \alpha_{i}\left[\frac{i+1}{k+1}\left(\begin{array}{l}
k+1 \\
i-k
\end{array}\right) a_{0} \alpha^{-i}+\frac{i}{k+1}\left(\begin{array}{c}
k+1 \\
i-k-1
\end{array}\right) a_{1} \alpha^{-i+1}\right. \\
+ & \left.\frac{i-1}{k+1}\left(\begin{array}{c}
k+1 \\
i-k-2
\end{array}\right) a_{2} \alpha^{-i+2}\right],
\end{aligned}
$$

and $V_{k}, X_{k}$ are obtained from $U_{k}, W_{k}$ be replacing

$$
a_{0}, a_{1}, a_{2}, \alpha \text { by } b_{0}, b_{1}, b_{2}, \beta \text {. }
$$

Let $k$ be arbitrary but fixed. If we divide (3.6) by $\alpha^{2 p+2 k+1}, \alpha^{2 p+2 k+2}$ respectively and let $p \rightarrow \infty$, then since $|\beta / \alpha|<1$ we get

$$
U_{k}=0, W_{k}=0 \quad(k=0,1, \cdots) .
$$

And from (3.6) we then have

$$
V_{k}=0, X_{k}=0 \quad(k=0,1, \cdots) .
$$

For $k=0,(3.8),(3.9)$ reduce to

$$
\begin{aligned}
& \alpha_{0} a_{0}+\alpha_{1} a_{1}+\alpha_{2} a_{2}=0, \alpha_{1} a_{0}+\alpha_{2} a_{1}+\alpha_{3} a_{2}=0, \\
& \alpha_{0} b_{0}+\alpha_{1} b_{1}+\alpha_{2} b_{2}=0, \alpha_{1} b_{0}+\alpha_{2} b_{1}+\alpha_{3} b_{2}=0 .
\end{aligned}
$$

Now from (3.3) with $p=k=0$ we have

$$
\alpha_{0} r_{1}+\alpha_{1} s_{1}+\alpha_{2} t_{1}=0 \text {. }
$$


But $r_{1}=t_{1}=0, s_{1}=1$; hence

$$
\alpha_{1}=0 \text {. }
$$

So (3.10) becomes

$$
\begin{aligned}
& \alpha_{0} a_{0}+\alpha_{2} a_{2}=0, \alpha_{2} a_{1}+\alpha_{3} a_{2}=0, \\
& \alpha_{0} b_{0}+\alpha_{2} b_{2}=0, \alpha_{2} b_{1}+\alpha_{3} b_{2}=0 .
\end{aligned}
$$

Now $a_{2} b_{2} \neq 0$. For if $a_{2}$ or $b_{2}$ is zero then from $a_{2} \alpha+b_{2} \beta=0$ (in (1.8)) and $\alpha \beta \neq 0$ we get $\alpha_{2}=b_{2}=0$. Hence (again from (1.8)) $c_{2}=$ 0 ; so all coefficients in $(1.3 b)$ are of degree $<2$, contrary to assumption. Again, $a_{0} b_{0} \neq 0$. For if $a_{0}$ or $b_{0}$ is zero then (3.11) implies that $\alpha_{2}=$ 0 . Since we already have $\alpha_{1}=0$, then $\Delta_{1}=\left|\begin{array}{l}\alpha_{0} \alpha_{1} \\ \alpha_{1} \alpha_{2}\end{array}\right|=0$. But for the moments $\left\{\alpha_{n}\right\}$ corresponding to an orthogonal set it is known [2] that

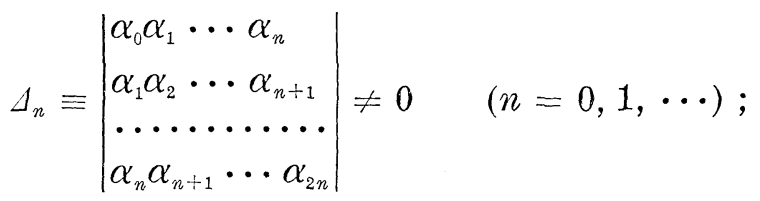

so we have a contradiction. Thus,

$$
a_{2} b_{2} \neq 0, a_{0} b_{0} \neq 0, \alpha_{2} \neq 0 \text {. }
$$

The right hand equations in (3.11) give us

$$
-b_{1} a_{2}+a_{1} b_{2}=0 \text {. }
$$

This with

$$
\alpha a_{2}+\beta b_{2}=0
$$

from (1.8) implies

$$
\alpha a_{1}+\beta b_{1}=0,
$$

contrary to (1.9) for $n=0$. So the assumption $\beta \neq-\alpha$ leads to a contradiction, and we have

$$
\beta=-\alpha
$$

Then from (1.12):

$$
a_{0}=b_{0}
$$

In (3.2) we now have

$$
\left\{\begin{array}{l}
r_{2 p}=2 a_{0} \delta^{-1} \alpha^{2 p}, s_{2 p}=\left(a_{1}+b_{1}\right) \delta^{-1} \alpha^{2 p}, t_{2 p}=\left(a_{2}+b_{2}\right) \delta^{-1} \alpha^{2 p} \\
r_{2 p+1}=0, s_{2 p+1}=\left(a_{1}-b_{1}\right) \delta^{-1} \alpha^{2 p+1}, t_{2 p+1}=\left(a_{2}-b_{2}\right) \delta^{-1} \alpha^{2 p+1}
\end{array}\right.
$$


$(p=1,2, \cdots)$, with $r_{1}=t_{1}=0, s_{1}=1$. (3.12) and (3.15) show that

$$
r_{2} \neq 0 \text {. }
$$

Let

$$
u_{p}=s_{2 p+1}, v_{p}=t_{2 p+1}, w_{p}=t_{2 p+2} .
$$

From $\alpha_{2} \alpha+b_{2} \beta=0, \beta=-\alpha \neq 0$ we get

$$
a_{2}=b_{2} \text {. }
$$

It is then readily seen that

$$
v_{p}=0, w_{p}-t_{2} u_{p}=0 \quad(p=0,1,2, \cdots)
$$

Choose $r_{2}, s_{2}, t_{2}, s_{3}$ to satisfy the conditions

$$
r_{2} \neq 0,2+k t_{2} \neq 0(k=0,1, \cdots), \Delta_{2} \neq 0,
$$

where $\alpha_{1}=0, \alpha_{2}, \alpha_{3}, \alpha_{4}$ are obtained from the equations

$$
D_{0}^{0}=0, d_{1}^{0}=0, D_{1}^{0}=0 .
$$

(3.18)-(3.20) make Theorem 4.2 ([1], p. 158) applicable, so that the solutions of $(1.3 \mathrm{~b})$ form a weak orthogonal set if and only if

$$
\left\{\begin{array}{l}
s_{2 p+1}=s_{3}^{p}, t_{2 p+1}=0, r_{2 p+1}=0 \\
s_{2 p+2}=s_{2} s_{3}^{p}, t_{2 p+2}=t_{2} s_{3}^{p}, r_{2 p+2}=r_{2} s_{3}^{p}
\end{array} \quad(p=0,1, \cdots) .\right.
$$

Now these conditions do hold in view of (3.14).

The first two conditions of (3.19) become

$$
a_{0} \neq 0 ;\left(a_{1}-b_{1}\right)+k \alpha a_{2} \neq 0 \quad(k=0,1,2, \cdots) .
$$

Finally, for weak orthogonality to imply orthogonality it is necessary and sufficient ([1], pp. 161-162) that $t_{2} \notin S\left(r_{2}, s_{2}, s_{3}\right)$ where $S\left(r_{2}, s_{2}, s_{3}\right)$ is the set of all real values of $t_{2}$ for which $\pi_{n}\left(r_{2}, s_{2}, s_{3}, t_{2}\right)=0$ for some $n>1$. The expression for $\pi_{n}$ is lengthy, and we do not reproduce it here. We merely observe that for given $r_{2}, s_{2}, s_{3}$ the set $S\left(r_{2}, s_{2}, s_{3}\right)$ is at most denumerable.

To sum up:

THEOREM 3.1. Let the admissible equation (1.3b) be canonical. Its solutions form an orthogonal polynomial set if and only if:

(i ) (3.12), (3.13), (3.14), (3.17), (3.19) hold.

(ii) $t_{2} \notin S\left(r_{2}, s_{2}, s_{3}\right)$.

REMARKs. (a ) If the canonical equation (1.3b) has an orthogonal polynomial set of solutions then it has the form 


$$
\begin{aligned}
\left(a_{2} x^{2}+a_{1} x+a_{0}\right) y(x+\alpha) & +\left(a_{2} x^{2}+b_{1} x+a_{0}\right) y(x-\alpha) \\
- & {\left[2 a_{2} x^{2}+\left(a_{1}+b_{1}\right) x\right] y(x)=\lambda y(x), }
\end{aligned}
$$

with

(3.24) $a_{0} a_{2}\left(a_{1}-b_{1}\right) \alpha \neq 0 ;\left(a_{1}-b_{1}\right)+k \alpha a_{2} \neq 0 \quad(k=0,1, \cdots)$.

(b) As in $\S 2$ the transformation $x=\alpha x^{*}, z\left(x^{*}\right)=y\left(\alpha x^{*}\right)$ carries (3.24) into a similar equation with $\alpha$ replaced by 1 .

4. Two examples. If an orthogonal polynomial set $\left\{P_{n}(x)\right\}$ satisfies (2.10) with $\lambda=\lambda_{n}$ for $y=P_{n}(x)$ then from (1.6) we have

$$
\lambda_{n}=2 a_{0}+n \alpha\left(a_{1}-b_{1}\right) \quad(n=0,1, \cdots) .
$$

Let $\left\{P_{n}(x)\right\},\left\{Q_{n}(x)\right\}$ be polynomial sets defined by the respective generating functions

$$
\begin{gathered}
e^{c t}(1-t)^{x+c}=\sum_{n=0}^{\infty} P_{n}(x) t^{n} \quad(c \neq 0), \\
(1-t)^{x-b d} \cdot(1-b t)^{-x+d}=\sum_{n=0}^{\infty} Q_{n}(x) t^{n} \quad(b \neq 0,1) .
\end{gathered}
$$

We shall show that these sets are orthogonal and satisfy an equation of the form (2.10).

Denote the left side of $(2.10)$ by $L[y]$. If $G(x, t)$ is the generating function in (4.2) then

$$
L[G]=G\left\{\left(a_{1} x+a_{0}\right)(1-t)^{\alpha}+\left(b_{1} x+a_{0}\right)(1-t)^{-\alpha}-\left(a_{1}+b_{1}\right) x\right\} .
$$

Also,

$$
\begin{aligned}
& \sum_{n=0}^{\infty} \lambda_{n} P_{n}(x) t^{n}=2 a_{0} G+\alpha\left(a_{1}-b_{1}\right) t \partial G / \partial t \\
& \quad=G\left\{2 a_{0}+\alpha\left(a_{1}-b_{1}\right) t\left[c-(x+c)(1-t)^{-1}\right]\right\} .
\end{aligned}
$$

$\left\{P_{n}(x)\right\}$ will satisfy (2.10) if (4.4) and (4.5) are identical. It is a straightforward computation to show that they are identical if

$$
\alpha=1 ; a_{1}=0 ; b_{1}=a_{0} / c .
$$

Hence $\left\{P_{n}(x)\right\}$ is an orthogonal set which satisfies the equation

$$
P_{n}(x+1)+(x+c) P_{n}(x-1)-x P_{n}(x)=(2 c-n) P_{n}(x) .
$$

In the same way it is found that $\left\{Q_{n}(x)\right\}$ is an orthogonal set that is a solution of (2.10) for

$$
\alpha=1 ; a_{1}=b b_{i} ; a_{n}=-b d b_{1} .
$$


The equation reduces to

$$
\begin{gathered}
b(x-d) Q_{n}(x+1)+(x-b d) Q_{n}(x-1)-(b+1) x Q_{n}(x) \\
=\{-2 b d+n(b-1)\} Q_{n}(x) .
\end{gathered}
$$

In the case of $(4.9)$ the condition $\left(2.9_{2}\right)$ is to hold. It reduces to

$$
-d(b-1) \neq 0,1,2, \cdots \text {. }
$$

\section{REFERENCES}

1. H. L. Krall and I. M. Sheffer, Differential equations of infinite order for orthogonal polynomials, Annali di Matematica (4) 74 (1966), 135-172.

2. G. Szegö, Orthogonal polynomials, American Mathematical Society Colloquium Publications, vol. 23, 1959.

Received March 12, 1968.

Pennsylvania State University 


\section{PACIFIC JOURNAL OF MATHEMATICS}

\section{EDITORS}

H. ROYDEN

Stanford University

Stanford, California

\section{R. $R$ PHELPS}

University of Washington

Seattle, Washington 98105

\section{J. DugunduI}

Department of Mathematics

University of Southern California

Los Angeles, California 90007

RICHARD ARENS

University of California

Los Angeles, California 90024

\section{ASSOCIATE EDITORS}

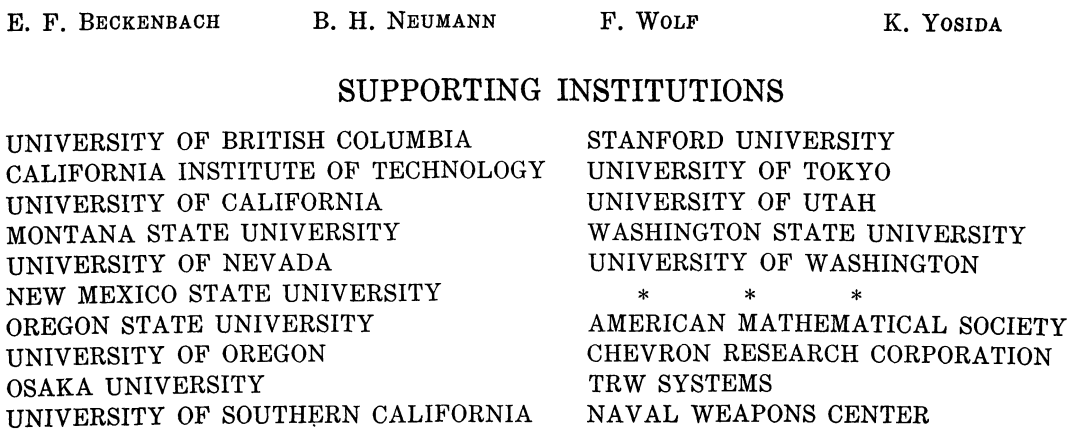

The Supporting Institutions listed above contribute to the cost of publication of this Journal, but they are not owners or publishers and have no responsibility for its content or policies.

Mathematical papers intended for publication in the Pacific Journal of Mathematics should be in typed form or offset-reproduced, double spaced with large margins. Underline Greek letters in red, German in green, and script in blue. The first paragraph or two must be capable of being used separately as a synopsis of the entire paper. It should not contain references to the bibliography. Manuscripts, in duplicate if possible, may be sent to any one of the four editors. Please classify according to the scheme of Math. Rev. 36, 1539-1546. All other communications to the editors should be addressed to the managing editor, Richard Arens, University of California, Los Angeles, California, 90024.

50 reprints are provided free for each article; additional copies may be obtained at cost in multiples of 50 .

The Pacific Journal of Mathematics is published monthly. Effective with Volume 16 the price per volume (3 numbers) is $\$ 8.00$; single issues, $\$ 3.00$. Special price for current issues to individual faculty members of supporting institutions and to individual members of the American Mathematical Society: $\$ 4.00$ per volume; single issues $\$ 1.50$. Back numbers are available.

Subscriptions, orders for back numbers, and changes of address should be sent to Pacific Journal of Mathematics, 103 Highland Boulevard, Berkeley, California, 94708.

PUBLISHED BY PACIFIC JOURNAL OF MATHEMATICS, A NON-PROFIT CORPORATION

Printed at Kokusai Bunken Insatsusha (International Academic Printing Co., Ltd.), 7-17, Fujimi 2-chome, Chiyoda-ku, Tokyo, Japan. 


\section{Pacific Journal of Mathematics}

Vol. 28, No. $2 \quad$ April, 1969

Richard Arens and Donald George Babbitt, The geometry of relativistic

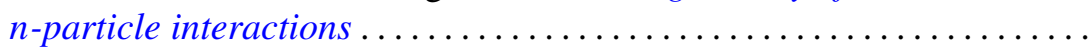

Kirby Alan Baker, Hypotopological spaces and their embeddings in lattices

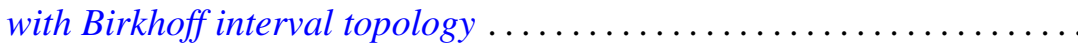

J. Lennart (John) Berggren, Finite groups in which every element is conjugate to its inverse ........................... 289

Beverly L. Brechner, Homeomorphism groups of dendrons . . . . . . . . . . . 295

Robert Ray Colby and Edgar Andrews Rutter, QF - 3 rings with zero singular ideal ................................. 303

Stephen Daniel Comer, Classes without the amalgamation property....... 309

Stephen D. Fisher, Bounded approximation by rational functions ......... 319

Robert Gaines, Continuous dependence for two-point boundary value problems..................................... 327

Bernard Russel Gelbaum, Banach algebra bundles ............... 337

Moses Glasner and Richard Emanuel Katz, Function-theoretic degeneracy criteria for Riemannian manifolds ...................... 351

Fletcher Gross, Fixed-point-free operator groups of order $8 \ldots \ldots \ldots \ldots 357$

Sav Roman Harasymiv, On approximation by dilations of distributions . . . . 363

Cheong Seng Hoo, Nilpotency class of a map and Stasheff's criterion ... . . 375

Richard Emanuel Katz, A note on extremal length and modutus.......... 381

H. L. Krall and I. M. Sheffer, Difference equations for some orthogonal polynomials ................................

Yu-Lee Lee, On the construction of lower radical properties ........... 393

Robert Phillips, Liouville's theorem........................... 397

Yum-Tong Siu, Analytic sheaf cohomology groups of dimension $n$ of

n-dimensional noncompact complex manifolds ..... . .

Michael Samuel Skaff, Vector valued Orlicz spaces. II...

James DeWitt Stein, Homomorphisms of $B^{*}$-algebras .... . .

Mark Lawrence Teply, Torsionfree injective modules .... . . .

Richard R. Tucker, The $\delta^{2}$-process and related topics. II .

David William Walkup and Roger Jean-Baptiste Robert Wets, Lifting

projections of convex polyhedra...

Thomas Paul Whaley, Large sublattices of a lattice. 